\title{
Investigation of Light Absorption in a ZnS Quantum Dot
}

\author{
Hojjatollah K. Salehani ${ }^{1}$ and Maedeh Zakeri ${ }^{2}$ \\ ${ }^{1}$ Department of Physics, Damavand Branch, Islamic Azad University, Moallem Square, Damavand 39718-78911, Iran \\ ${ }^{2}$ Young Researchers and Elites Club, Damavand Branch, Islamic Azad University, Moallem Square, Damavand 39718-78911, Iran
}

Correspondence should be addressed to Hojjatollah K. Salehani; hojjat.salehani@gmail.com

Received 4 May 2013; Accepted 16 July 2013

Academic Editor: Mircea Cotlet

Copyright (c) 2013 H. K. Salehani and M. Zakeri. This is an open access article distributed under the Creative Commons Attribution License, which permits unrestricted use, distribution, and reproduction in any medium, provided the original work is properly cited.

\begin{abstract}
The light absorption of a $\mathrm{ZnS}$ quantum dot with a parabolic confinement potential is studied in this paper in the presence of magnetic field perpendicular to dot plane. The Schrodinger equation of a single electron is solved numerically, and energy spectra and wave functions are obtained. Then, the optical absorption coefficients in transition from ground state to different excited states are calculated. The effects the magnetic field and quantum dot width on the optical absorption are investigated. It is found that the optical absorption coefficient has a blue shift by increasing of magnetic field or confinement strength of quantum dot.
\end{abstract}

\section{Introduction}

During the last ten years, with great progress in nanofabrication techniques, it is possible to grow semiconductor quantum dots (QDs) with various shapes and sizes [1]. QDs are semiconductor nanocrystals that can be considered as artificial atoms because they have discrete energy levels and shell structure [2]. The main advantage of QD relative to real atom is that the physical properties of QDs can be controlled by tuning their size and shapes. The QDs have various applications; in particular in the mid- and far-infrared regions, they can be used for pollution detection, thermal imaging object location, and remote sensing as well as infrared imaging of astronomical objects [3]. This nanostructure can be formed by modern growth methods such as molecular beam epitaxy [4] and the Stranski-Krastanov growth method $[5,6]$.

Recently, the synthetic chrysotile nanotubes have been synthesized by Roveri et al. [7]. These geoinspired nanotubes can be prepared with specific properties, finalized to focused achievements such as preparation of new quantum wires. On the other hand, an experimental study is carried out on assembling ZnS QDs in synthetic chrysotile nanotubes [8]. These assembling QDs affect significantly the optical properties of the synthetic chrysotile nanotube. Due to potential application of these nanotubes as nonlinear optical and conducting technological devices, investigation of the electronic and optical properties of these systems is essential. In this paper, we investigate the light absorption in a ZnS QD. The effects of magnetic field and geometrical size of ZnS QD on the light absorption are investigated theoretically.

Thus far, the optical properties of QDs are studied by some authors [9-13]. The first study of optical absorption in QD has been carried out theoretically by Efros and his colleague [9]. They have investigated the peculiarities of interband optical absorption in spherical QD. In [10], the authors have studied the light absorption in a cylindrical QD. In the presence of the electric and magnetic fields, the interband light absorption of parabolic QD has been investigated theoretically [11]. The electron states and direct interband absorption of light in a strongly prolate ellipsoidal QD are studied at three regimes [12]. However, as far as we know, the effect of QD size on the light absorption of a ZnS parabolic QD is not investigated. The purpose of the present paper is to study the geometrical size and magnetic field effects on the optical properties of a ZnS QD. Therefore, we solve the Schrodinger equation of a single electron; and the energy spectra and absorption coefficients of parabolic QD are obtained. This paper is organized as follows. In Section 2, we present the theoretical model. In Section 3, the results of a numerical study are presented and discussed. Finally, Section 4 ends the paper with conclusion. 


\section{Theoretical Model}

Consider a two-dimensional QD in a $x-y$ plane. The confinement potential of the QD is considered to be parabolic; is shown schematically in Figure 1, and can be written as

$$
V_{c}(r)=\frac{1}{2} \mu \omega^{2} r^{2}
$$

where $\mu$ is the effective mass of electron, $\omega$ is the confinement potential strength, and $r$ is the radial distance of origin.

In the presence of a magnetic field perpendicular on QD plane, the Hamiltonian of a single electron can be written as

$$
\begin{aligned}
H= & \frac{\hbar^{2}}{2 \mu}\left[-\frac{\partial^{2}}{\partial r^{2}}-\frac{1}{r} \frac{\partial}{\partial r}-\frac{1}{r^{2}} \frac{\partial^{2}}{\partial \varphi^{2}}-\frac{e B i \partial}{\hbar \partial \varphi}+\frac{e^{2} B^{2} r^{2}}{4 \hbar^{2}}\right] \\
& +V_{c}(r)
\end{aligned}
$$

where $i$ is a square root of -1 (i.e., $i=\sqrt{-1}$ ), $h=2 \pi \hbar$ is the Planck constant, $-e$ is the charge of electron, $B$ is the magnetic field, and $r$ and $\varphi$ are, respectively, the radial and angular coordinates of polar coordinates system. The electron wave function in a QD with circular symmetry can be written as

$$
\psi_{n L}(r, \varphi)=\frac{1}{\sqrt{2 \pi}} e^{i L \varphi} R_{n}(r), \quad L=0, \pm 1, \pm 2, \ldots,
$$

where $n$ and $L$ are radial and angular quantum numbers, respectively. Also, $R_{n}(r)$ is $n$th radial wave function. Operating on the previous wavefunction (see (3)) with the Hamiltonian (see (2)) gives the following Schrodinger equation:

$$
\begin{gathered}
\frac{\hbar^{2}}{2 \mu}\left[-\frac{\partial^{2}}{\partial r^{2}}-\frac{1}{r} \frac{\partial}{\partial r}-\frac{L^{2}}{r^{2}}-\frac{e B L}{\hbar}+\frac{e^{2} B^{2} r^{2}}{4 \hbar^{2}}\right] R(r) \\
+V_{c}(r) R(r)=E R(r),
\end{gathered}
$$

where $E$ is energy of a single electron.

\section{Results and Discussion}

In this section, we present the results of a numerical study based on equations derived in the previous section. The Schrodinger equation (see (4)) is solved numerically using the finite difference method and exact diagonalization technique [14]. The energy spectra and eigenstates are obtained. In general, the optical absorption coefficient for a transition from initial state $\left|n^{\prime}, L^{\prime}\right\rangle$ to final state $|n, L\rangle$ is calculated as [15]

$$
\alpha(\nu)=\sum_{n L}\left\langle n^{\prime}, L^{\prime}|\mathbf{e} \cdot \mathbf{r}| n, L\right\rangle \delta\left(E_{n L}-E_{n^{\prime} L^{\prime}}-h \nu\right),
$$

where $n^{\prime}$ and $L^{\prime}$ are the radial and angular quantum numbers of initial state and $n$ and $L$ are also the radial and angular quantum numbers of final state, respectively. The unit vector e indicates the direction of polarization of light. For in-plane polarization, the elements of transition matrix can be written as

$$
\begin{aligned}
& \left\langle n^{\prime}, L^{\prime}|\mathbf{e} \cdot \mathbf{r}| n, L\right\rangle \\
& \quad=\frac{1}{2 \pi}\left(e_{x}+i e_{y}\right) \delta_{\left|L-L^{\prime}\right| 1} \int_{0}^{\infty} d r r^{2} R_{n^{\prime} L^{\prime}}(r) R_{n L}(r) .
\end{aligned}
$$

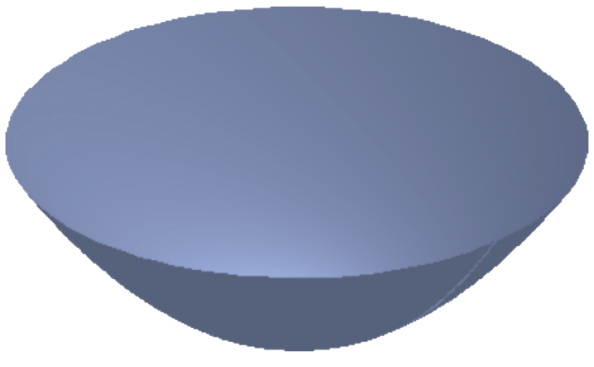

FIGURE 1: Schematic view of a parabolic confinement potential for a two-dimensional QD.

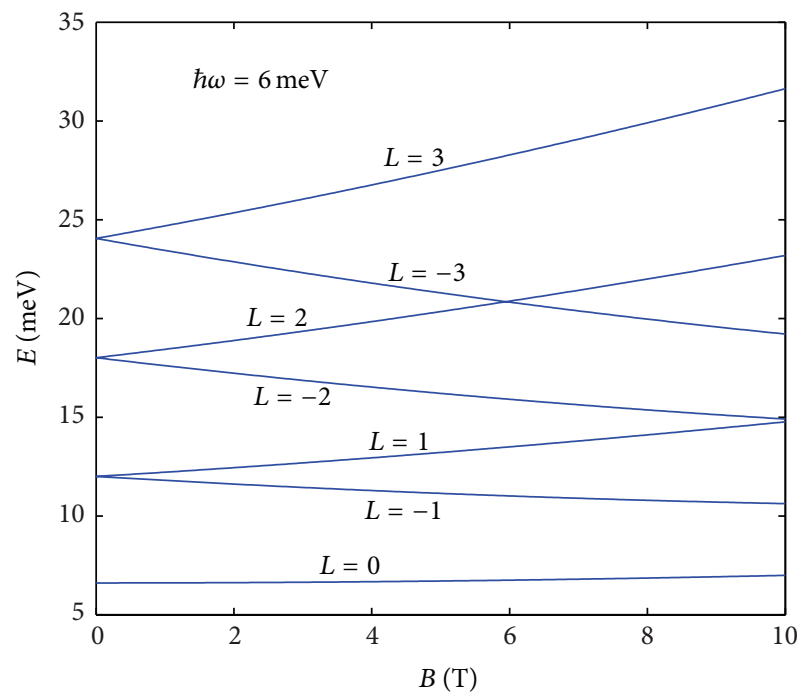

FIGURE 2: The energy spectrum of a single electron as a function of magnetic field for different quantum numbers of angular momentum.

It is clear that the elements of transition matrix are not equal zero when $\delta_{\left|L-L^{\prime}\right| 1} \neq 0$ (i.e., $L-L^{\prime}= \pm 1$ ). For numerical calculation, the electron effective mass is taken to be $m^{*}=$ $0.28 m_{o}$ ( $\mathrm{ZnS}$ value) where $m_{o}$ is the free electron mass. The energy spectrum of a single electron is shown in Figure 2 as a function of magnetic field for different angular momentum quantum numbers. This is in agreement with the result of [16] which provides an analytical expression for energy eigenvalue. It is observed that for the two lowest energy levels there are not any angular momentum transition in this range of magnetic field. In the absence of magnetic field (i.e., $B=$ 0 ), energy levels with symmetric angular momentums are degenerate.

In the absence of magnetic field, the optical absorption coefficients for transition from ground state (i.e., $\mid n^{\prime}=1$, $\left.L^{\prime}=0\right\rangle$ ) to the different excited states (i.e., $|n=1, l= \pm 1\rangle$, $|n=2, l= \pm 1\rangle,|n=3, l= \pm 1\rangle)$ are calculated in a QD with $\hbar \omega=6 \mathrm{meV}$ and are shown in Figure 3 as a function of incident photon energy. It can be observed that the main peak corresponds to the transition from ground state (i.e., $\left.\left|n^{\prime}=1, L^{\prime}=0\right\rangle\right)$ to $|n=1, L= \pm 1\rangle$ while for the other transitions the peak value decreases at least a thousand 


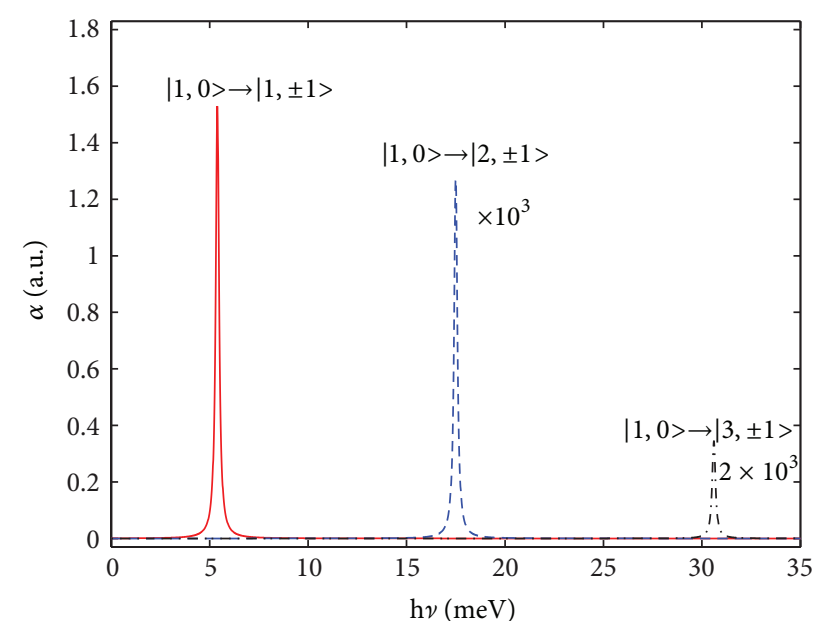

FIGURE 3: The optical absorption coefficients for transition from the ground state to the excited states as a function of incident photon energy for $\hbar \omega=6 \mathrm{meV}$ and $B=0$.

times. These results reveal that, in the experimental study of intraband spectra, the transition from ground state (i.e., $\left.\left|n^{\prime}=1, L^{\prime}=0\right\rangle\right)$ to $|n=1, L= \pm 1\rangle$ states is remarkable and it is expected to be easily observed. The effect of magnetic field on the absorption coefficient is also calculated and shown in Figure 4. In this figure, the absorption coefficients are shown for transition from ground state (i.e., $\mid n^{\prime}=1$, $\left.\left.L^{\prime}=0\right\rangle\right)$ to excited states $|n=1, L= \pm 1\rangle$ for $B=$ $0,2 T$ and $4 T$ as functions of incident photon energy. It can be observed that the magnetic field does not affect the peak value of the absorption coefficient, but increasing the magnetic field causes the QD to absorb photons with higher energies. To investigate the effect of QD size on the light absorption, we calculate the absorption coefficient for different confinement strengths. The confinement strength corresponds to the QD width via confinement potential (see (1)). In other words, according to the mentioned equation, increasing the confinement strength decreases the QD width and vice versa. Figure 5 shows the absorption coefficients for transition from ground state (i.e., $\left|n^{\prime}=1, L^{\prime}=0\right\rangle$ ) to $|n=1, L= \pm 1\rangle$ states for different confinement strengths of the QD as a function of the incident photon energy. It can be observed that the peak value decreases when the QD width is decreased. In addition, it is observed that a wider QD absorbs the photons with lower energies.

\section{Conclusion}

We have presented a theoretical study of light absorption in a two-dimensional ZnS QD. The absorption coefficients in transition from ground state to different excited states are calculated. The effects of magnetic field and QD width on the absorption coefficient are investigated. In the absence of magnetic field, it is found that the main peak corresponds to the transition from ground state to first excited state while for the other transitions the peak value is decreased at least a thousand times. Increasing of the applied magnetic field

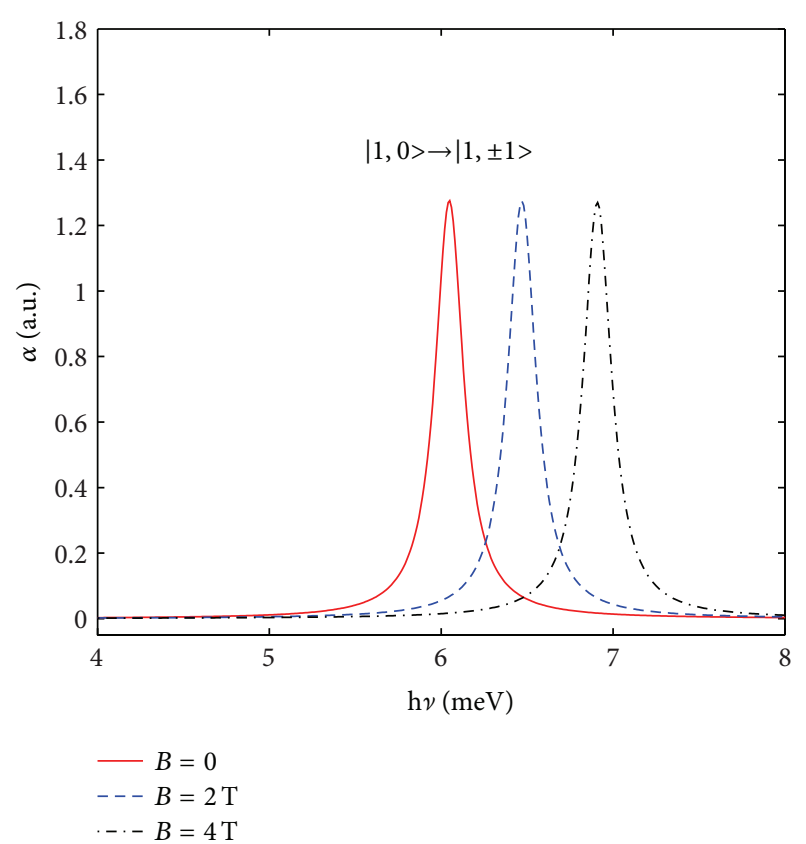

FIGURE 4: The optical absorption coefficients for transition from ground state to the excited states as a function of the incident photon energy for $\hbar \omega=6 \mathrm{meV}$ and different magnetic fields.

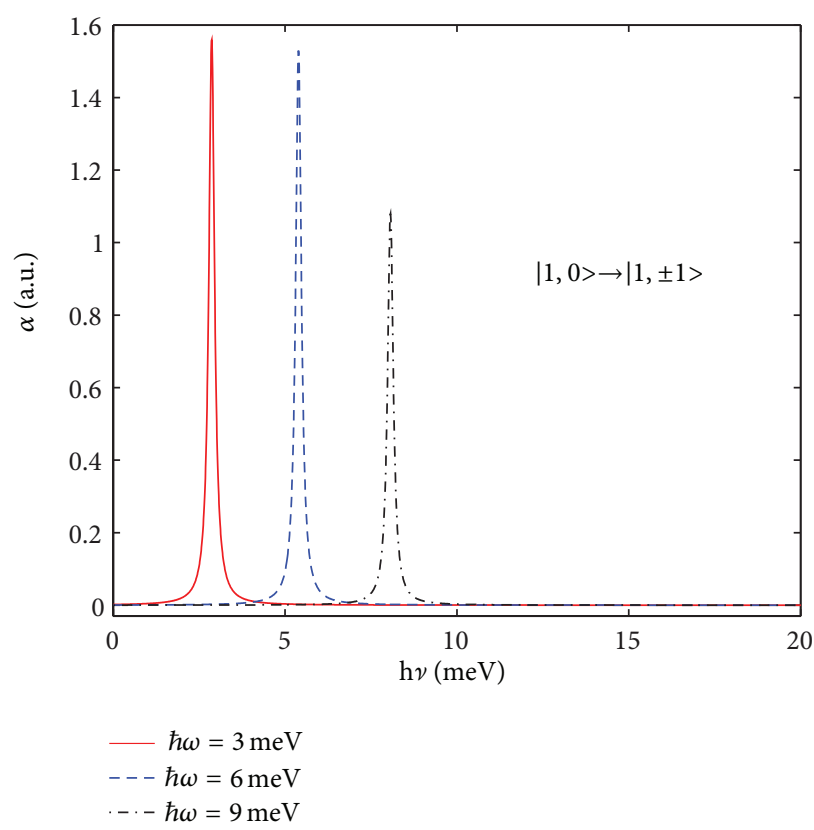

Figure 5: The optical absorption coefficients as a function of the incident photon energy for $B=0$ and different confinement strengths.

or the QD confinement strength causes the QD to absorb photons with higher energies.

\section{References}

[1] P. Harrison, Quantum Wells, Wires and Dots, Theoretical and Computational Physics, John Wiley \& Sons, New York, NY, USA, 2005. 
[2] D. Gammon, "Semiconductor physics: electrons in artificial atoms," Nature, vol. 405, pp. 899-900, 2000.

[3] P. M. Petroff and N. Horiguchi, "Mid infrared and near infrared light upconverter using self-assembled quantum dots," U.S. Patent 6, 541, 788, 2003.

[4] S. Suraprapapich, S. Thainoi, S. Kanjanachuchai, and S. Panyakeow, "Self-assembled quantum-dot molecules by molecular-beam epitaxy," Journal of Vacuum Science and Technology $B$, vol. 23, no. 3, pp. 1217-1220, 2005.

[5] P. J. Carrington, V. A. Solov'ev, Q. Zhuang, S. V. Ivanov, and A. Krier, "InSb quantum dot LEDs grown by molecular beam epitaxy for mid-infrared applications," Microelectronics Journal, vol. 40, no. 3, pp. 469-472, 2009.

[6] C. Bru-Chevallier, A. El Akra, D. Pelloux-Gervais et al., "InGaAs quantum dots grown by molecular beam epitaxy for light emission on Si substrates," Journal of Nanoscience and Nanotechnology, vol. 11, no. 10, pp. 9153-9159, 2011.

[7] N. Roveri, G. Falini, E. Foresti, G. Fracasso, I. G. Lesci, and P. Sabatino, "Geoinspired synthetic chrysolite nanotubes," Journal of Materials Research, vol. 21, no. 11, pp. 2711-2725, 2006.

[8] P. T. Jiang, M. G. Hua, J. Y. Feng, and L. Tao, "An experimental study of assembling ZnS quantum-dots in synthetic chrysotile nano-tubes," Acta Petrologica et Mineralogica, vol. 26, p. 487, 2007.

[9] A. L. Efros and A. L. Efros, "Interband absorption of light in a semiconductor sphere," Soviet Physics. Semiconductors, vol. 16, no. 7, pp. 772-775, 1982.

[10] K. G. Dvoyan, D. B. Hayrapetyan, E. M. Kazaryan, and A. A. Tshantshapanyan, "Electronic states and light absorption in a cylindrical quantum dot having thin falciform cross section," Nanoscale Research Letters, vol. 4, no. 2, pp. 130-137, 2009.

[11] M. S. Atoyan, E. M. Kazaryan, and H. A. Sarkisyan, "Interband light absorption in parabolic quantum dot in the presence of electrical and magnetic fields," Physica E, vol. 31, no. 1, pp. 8385, 2006.

[12] K. G. Dvoyan, D. B. Hayrapetyan, and E. M. Kazaryan, "Direct interband light absorption in strongly prolated ellipsoidal quantum dots' ensemble," Nanoscale Research Letters, vol. 4, no. 2, pp. 106-112, 2009.

[13] N. A. . Hamizi and M. Johan, "Optical properties of CdSe quantum dots via non-TOP based route," International Journal of Electrochemical Science, vol. 7, pp. 8458-8467, 2012.

[14] S. Wu and L. Wan, "Electronic structures in a CdSe spherical quantum dot in a magnetic field: diagonalization method and variational method," Journal of Applied Physics, vol. 111, no. 6, Article ID 063711, 10 pages, 2012.

[15] R. P. A. Lima and M. Amado, "Electronic states of on- and off-center donors in quantum rings of finite width," Journal of Luminescence, vol. 128, no. 5-6, pp. 858-861, 2008.

[16] J. Kainz, S. A. Mikhailov, A. Wensauer, and U. Rossler, "Quantum dots in high magnetic fields: calculation of ground-state properties," Physical Review B, vol. 65, no. 11, Article ID 115305, 5 pages, 2002. 

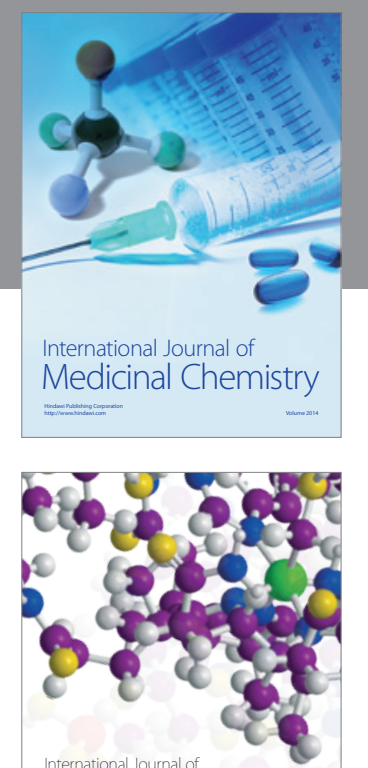

\section{Carbohydrate} Chemistry

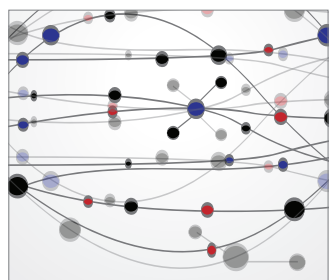

The Scientific World Journal
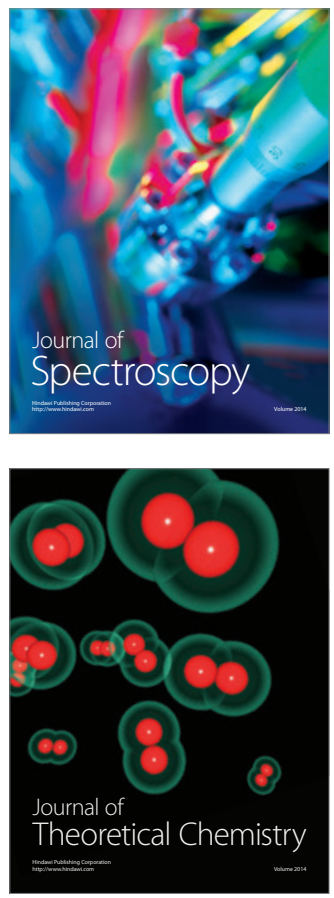
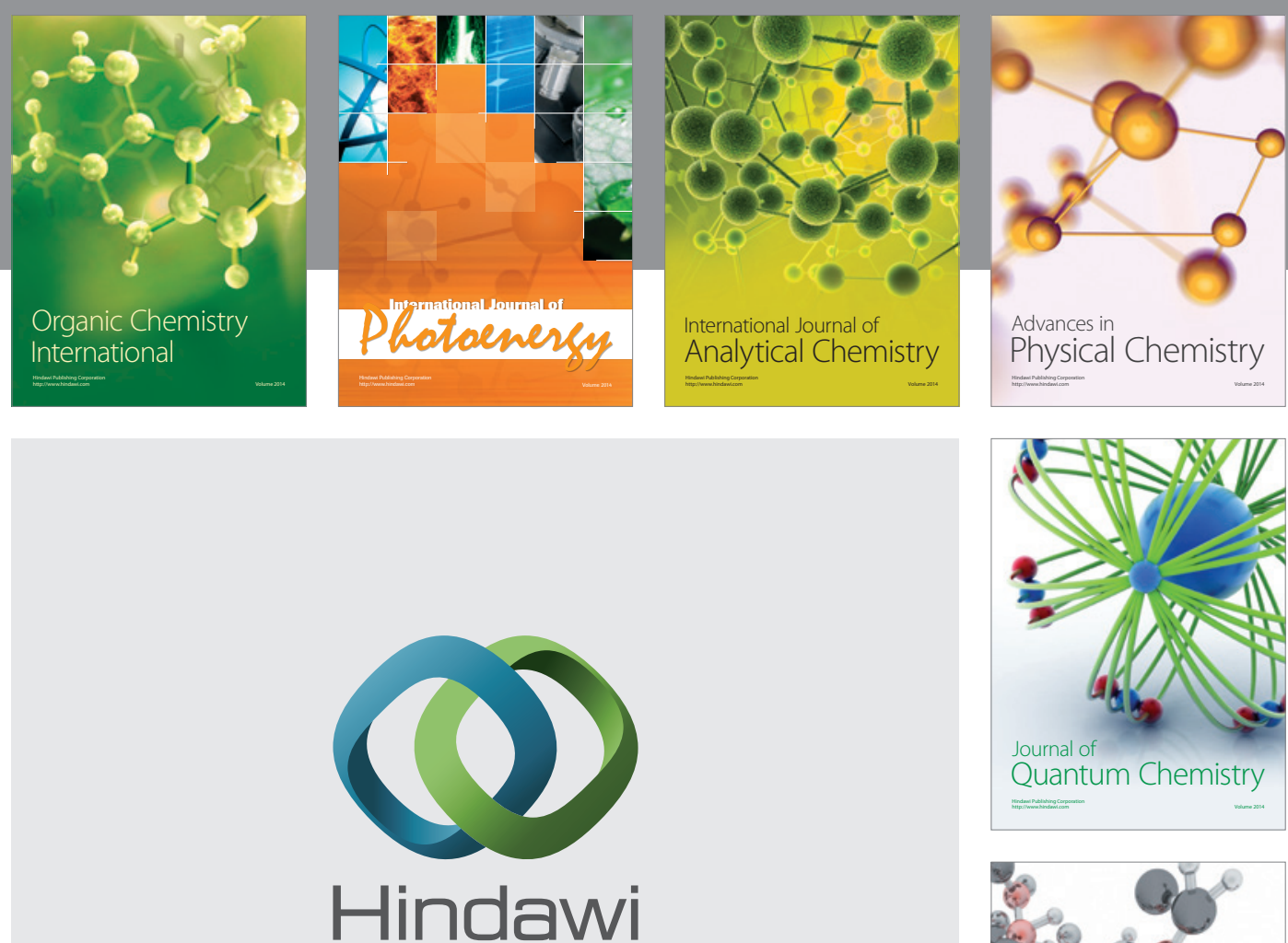

Submit your manuscripts at

http://www.hindawi.com

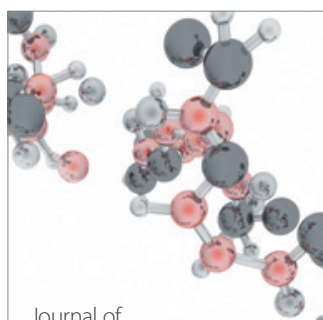

Analytical Methods

in Chemistry

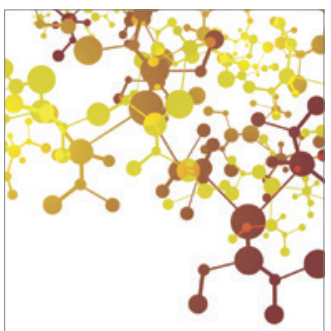

Journal of

Applied Chemistry

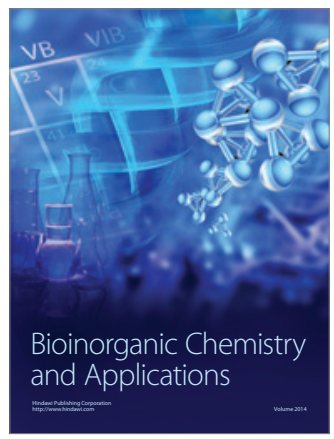

Inorganic Chemistry
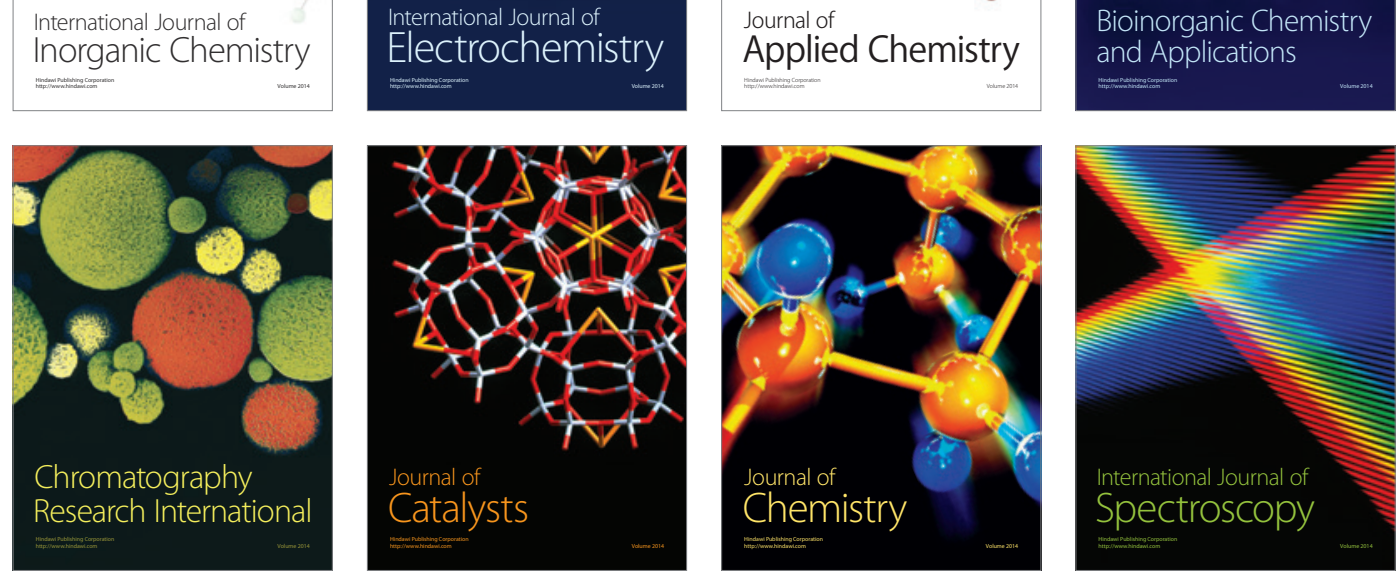